\title{
Differential signature of fecal microRNAs in patients with pancreatic cancer
}

\author{
YAN REN*", JUN GAO*, JIAN-QIANG LIU*, XIAO-WEI WANG, JUN-JUN GU, \\ HAO-JIE HUANG, YAN-FANG GONG and ZHAO-SHEN LI
}

\begin{abstract}
Department of Gastroenterology, Changhai Hospital, The Second Military Medical University, Shanghai 200433, P.R. China
\end{abstract}

Received October 14, 2011; Accepted January 10, 2012

DOI: $10.3892 / \mathrm{mmr} .2012 .862$

\begin{abstract}
The potential value of microRNAs as new biomarkers for pancreatic cancer (PCa) screening was explored in this study. Fecal microRNAs from stool samples obtained from 29 PCa patients, 22 chronic pancreatitis (CP) patients and 13 normal individuals were extracted, and 7 microRNAs (miR-16, miR-21, miR-155, miR-181a, miR-181b, miR-196a and miR-210) were detected. miR-181b and miR-210 discriminated PCa from normal individuals with receiver operating characteristic (ROC) curves and area under curve (AUC-ROC) of 0.745 and 0.772 , respectively. There was a significant correlation between miR-196a and the maximum tumor diameter (Spearman $\mathrm{r}=0.516, \mathrm{P}=0.041$ ). These findings suggest that fecal microRNAs such as miR-181b and miR-210 may have potential to be used as new biomarkers for PCa screening.
\end{abstract}

\section{Introduction}

Pancreatic cancer (PCa), which affects a dramatically increasing number of patients in recent years, is a highly malignant tumor with few treatment options available for patients with the disease. It is notorious for its late presentation, early and aggressive local invasion, metastatic potential and poor outcome (1). Only $20 \%$ of PCas are amenable to surgical resection at presentation (2) and despite the medical advances made over the last 20 years, PCa appears to have benefited the least in terms of survival. The main approach to improved prognosis of $\mathrm{PCa}$ is to develop new screening methods and identify novel biomarkers for the early detection of PCa.

microRNAs are a class of small non-coding RNAs encoded in the genomes of animals and plants (3-6), which play an important role in targeting messages of protein-coding

Correspondence to: Dr Zhao-Shen Li, Department of Gastroenterology, Shanghai Hospital, The Second Military Medical University, Shanghai 200433, P.R. China

E-mail: lizhaoshen111@gmail.com

*Contributed equally

Key words: fecal microRNAs, pancreatic cancer, diagnosis genes for cleavage or translational repression (7-9). The active microRNA products, $\sim 22 \mathrm{nt}$ in length, are formed from larger 60-110 nt hairpin precursor transcripts that serve as substrates for the double-stranded RNA endoribonuclease dicer (8). Recent studies indicate that microRNAs are mechanistically involved in the development of various human malignancies, suggesting that they represent a promising new class of cancer biomarkers. One of the most exciting biological features of microRNAs compared with mRNA is that they present a very stable form in different clinical samples such as serum, plasma, urine, and stool, because their small sizes are well protected from endogenous degradation (10-12). Recent studies have revealed that the expression patterns of microRNAs are a richer source of pathognomonic tumor information as compared with mRNA expression profiles (13). Furthermore, the expression patterns of microRNAs are unique to each tumor type and to their tissue of origin (13-19). Recently, several microRNAs in urinary samples of bladder cancer patients (20) and in the stool of colorectal cancer patients (21) were investigated to assess their diagnostic value. These findings raise the possibility that microRNAs in the stool may be used as novel non-invasive molecular markers for the early detection of PCa. However, there has been no report that explores this possibility. Therefore, the present study was carried out to determine whether fecal microRNAs can be used as biomarkers for early PCa diagnosis.

\section{Materials and methods}

Clinical samples. A total of 51 patients with $\mathrm{PCa}(\mathrm{n}=29)$ or chronic pancreatitis (CP) $(n=22)$ admitted to Changhai Hospital affiliated to The Second Military Medical University from 2008 to 2010 were included in this study. Each stool sample $(200 \mathrm{mg}$ ) was homogenized with buffer RPL (E.Z.N.A. RNA Stool kit; Omega, Guangzhou, China) and then kept at $-80^{\circ} \mathrm{C}$ until used for the detection of microRNAs. Patient clinicopathological data included age, gender, weight, smoking index, pancreatic ductal diameter, fasting blood glucose, serum levels of carbohydrate antigen 19-9 (CA19-9), carcinoembryonic antigen (CEA), alanine aminotransferase (ALT), aspartate aminotransferase (AST), $\gamma$-glutamyl transpeptidase (r-GT), bilirubin, leukocyte (WBC), neutrophils, and clinical stage. The diagnosis of PCa was confirmed based on histological 
Table I. Clinical characteristics of the patients with pancreatic cancer (PCa), those with chronic pancreatitis (CP) and normal subjects.

\begin{tabular}{|c|c|c|c|}
\hline & $\mathrm{PCa}(\mathrm{n}=29)$ & $\mathrm{CP}(\mathrm{n}=22)$ & Normal $(n=13)$ \\
\hline Age (years) mean $\pm \mathrm{SD}$ & $62.9 \pm 11.1$ & $47.2 \pm 11.8$ & $58.3 \pm 10.7$ \\
\hline \multicolumn{4}{|l|}{ Gender } \\
\hline Male & 19 & 15 & 8 \\
\hline Female & 10 & 7 & 5 \\
\hline \multicolumn{4}{|l|}{ Weight (kg) } \\
\hline$\geq 60$ & 13 & 12 & 4 \\
\hline$<60$ & 16 & 10 & 9 \\
\hline Smoking index (cigarettes/year) & $566.7 \pm 196.6$ & $474.1 \pm 351.1$ & $512.6 \pm 187.2$ \\
\hline \multicolumn{4}{|l|}{ White blood cell count (/liter) } \\
\hline$\geq 10 \times 10^{9}$ & 2 & 1 & 0 \\
\hline$<10 \times 10^{9}$ & 27 & 21 & 13 \\
\hline \multicolumn{4}{|l|}{ Neutrophils (/liter) } \\
\hline$\geq 7 \times 10^{9}$ & 2 & 1 & 0 \\
\hline$<7 \times 10^{9}$ & 27 & 21 & 13 \\
\hline \multicolumn{4}{|l|}{ Fasting blood glucose (mmol/l) } \\
\hline$\geq 6.1$ & 11 & 3 & 0 \\
\hline$<6.1$ & 18 & 19 & 13 \\
\hline \multicolumn{4}{|l|}{ ALT (U/l) } \\
\hline$\geq 40$ & 17 & 5 & 0 \\
\hline$<40$ & 12 & 17 & 13 \\
\hline \multicolumn{4}{|l|}{$\operatorname{AST}(\mathrm{U} / 1)$} \\
\hline$\geq 40$ & 15 & 5 & 0 \\
\hline$<40$ & 14 & 17 & 13 \\
\hline \multicolumn{4}{|l|}{ r-GT (U/l) } \\
\hline$\geq 50$ & 16 & 5 & 0 \\
\hline$<50$ & 13 & 17 & 13 \\
\hline \multicolumn{4}{|l|}{ Bilirubin $(\mu \mathrm{mol} / \mathrm{l})$} \\
\hline$<17.1$ & 13 & 6 & 0 \\
\hline$\geq 17.1$ & 16 & 16 & 13 \\
\hline \multicolumn{4}{|l|}{ CA19-9 (U/1) } \\
\hline$\geq 37$ & 22 & 6 & 0 \\
\hline$<37$ & 7 & 16 & 13 \\
\hline \multicolumn{4}{|l|}{$\mathrm{CEA}(\mathrm{ng} / \mathrm{ml})$} \\
\hline$\geq 10$ & 10 & 0 & 0 \\
\hline$<10$ & 19 & 22 & 13 \\
\hline Pancreatic ductal diameter $(\mathrm{cm})$ & $0.53 \pm 0.13$ & $0.71 \pm 0.34$ & \\
\hline Clinical stage (I-II/III-V) & $10 / 19$ & & \\
\hline
\end{tabular}

ALT, alanine aminotransferase; AST, aspartate aminotransferase; r-GT, glutamyl transpeptidase; CA19-9, carbohydrate antigen 19-9; CEA, carcinoma embryonic antigen.

examination of surgically resected tissue specimens $(n=2)$, and unequivocal histo/cytopathologic examination of EUS-FNAB $(\mathrm{n}=14)$, or the clinical integrated diagnostics criteria (such as clinical course, diagnostic imaging, serum CEA and CA19-9) combined with a long-term follow-up (ranging from 3 to 19 months) ( $n=13)$. The diagnoses of CP were confirmed based on imaging features, clinical integrated diagnostic criteria and long-term follow-up. Definitions and criteria in this article followed the General Rules for the Study of PCa, published by the Japan Pancreas Society (22).

In addition, the stool samples obtained from 13 healthy staff were used as a normal control. All patients and healthy 

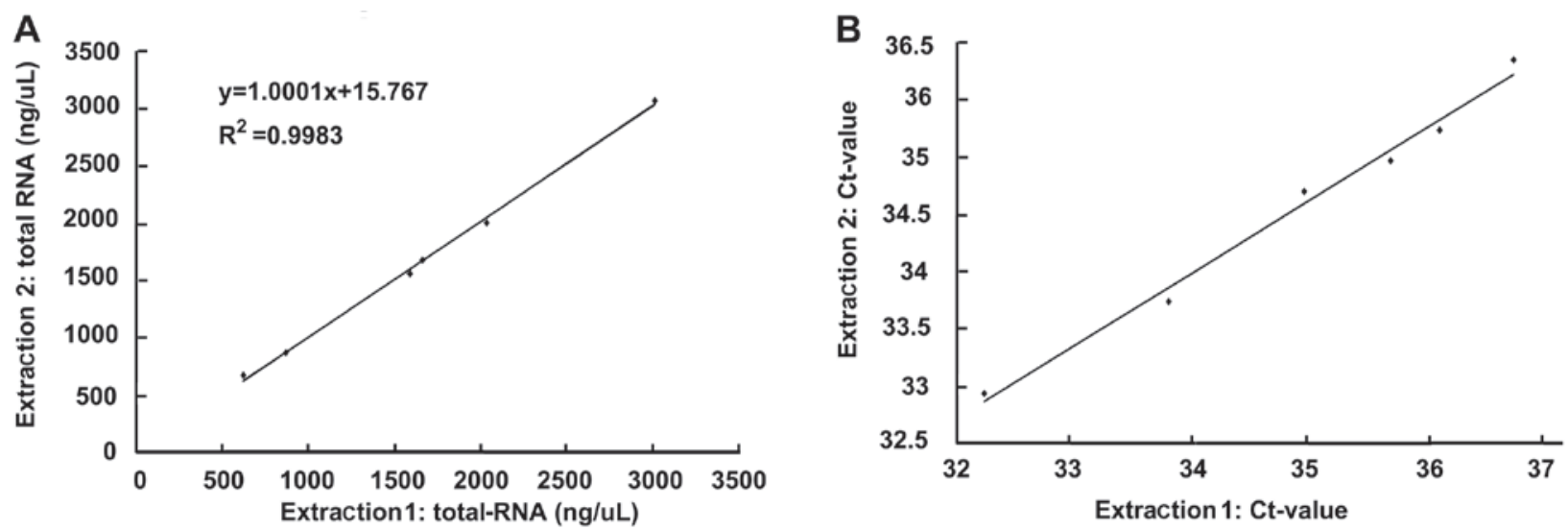

Figure 1. (A) Reproducibility of the methodology for extraction and detection of fecal total-RNA and (B) microRNAs in six stool samples at six-month intervals. A high linear correlation was noted in the amounts of fecal total-RNA $\left(\mathrm{R}^{2}=0.998, \mathrm{P}<0.001\right)$ and miR-16 $\left(\mathrm{R}^{2}=0.988, \mathrm{P}<0.001\right)$ between the two independent extraction procedures in the six samples.

volunteers provided written informed consent, and the study was approved by the Institutional Review Board.

RNA isolation. During the study time, $200 \mathrm{mg}$ of stool was collected from each of the subjects and homogenized with buffer RPL and then stored at $-80^{\circ} \mathrm{C}$ in a freezer. Then, the stored stool samples were used for extraction of total-RNA (including microRNAs) by using the E.Z.N.A. stool RNA kit, according to the manufacturer's instructions and performed as described previously (23). Briefly, an equal volume of acidic phenol, chloroform was added to the homogenized samples, and the aqueous phase was loaded onto the HiBind RNA membrane after the addition of the buffer RB and absolute ethanol. Finally, RNA was finally eluted in $100 \mu \mathrm{l}$ buffer AE. The concentrations of all RNA samples were quantified by the NanoDrop ND-1000 (NanoDrop, Wilmington, DE, USA).

microRNA selection and detection. Six microRNAs (miR-21, miR-155, miR-181a, miR-181b, miR-196a and miR-210) know to be related to PCa were selected to detect the relative abundance in the stool due to two previous studies $(24,25)$, while miR-16 was conjectured as a normalizer according to Link et al (21).

Quantification of microRNAs was performed using TaqMan microRNA assay (Applied Biosystems, CA, USA). Briefly, $1 \mu 1$ total-RNA was mixed with the TaqMan microRNA Reverse Transcription kit (Applied Biosystems), which contained $1 \mu 1 \mathrm{RNA}, 1 \mathrm{mmol} / \mathrm{l}$ of each deoxyribonucleotide triphosphate, 50 units of MultiScribe reverse transcriptase, $1 \mathrm{X}$ reaction buffer, 4 units RNase inhibitor, and 1X gene-specific primer, and then nuclease-free $\mathrm{H}_{2} \mathrm{O}$ was added to a final volume of $15 \mu \mathrm{l}$. The reaction program was at $16^{\circ} \mathrm{C}$ for $30 \mathrm{~min}$, followed by an incubation step at $42^{\circ} \mathrm{C}$ for $30 \mathrm{~min}$. The enzyme was inactivated at $85^{\circ} \mathrm{C}$ for $5 \mathrm{~min}$. Then, $2 \mu \mathrm{l}$ of the cDNA solution was amplified using $10 \mu 1$ TaqMan Universal PCR Master Mix (Applied Biosystems), $1 \mu$ l gene-specific primers/probe, and nuclease-free $\mathrm{H}_{2} \mathrm{O}$ in a final volume of $20 \mu \mathrm{l}$. The quantitative PCR was run on a 7500 HT quantitative PCR machine (Applied Biosystems) using a two-step PCR protocol with an initial denaturation step at $95^{\circ} \mathrm{C}$ for $10 \mathrm{~min}$, followed by 55 cycles with a denaturation step at $95^{\circ} \mathrm{C}$ for $15 \mathrm{sec}$, and an annealing/ elongation step at $60^{\circ} \mathrm{C}$ for $60 \mathrm{sec}$. The cycle threshold $(\mathrm{Ct})$ values were calculated with the SDS 2.1 software (Applied Biosystems). All reactions were run in triplicate.

The reliability of the normalizer, miR-16, was validated by Genorm software (18), which is able to determine the most stable gene expressed after input of all Ct-value data of the participant genes. The pool of fecal total-RNAs including microRNAs from 10 normal subjects which were selected from the 13 healthy staff members was referred as the control for calculation. The relative microRNA abundance was determined as below:

Relative microRNA abundance $(-\Delta \Delta \mathrm{Ct})=-\left[\left(\mathrm{Sample}_{\mathrm{Ct}} \mathrm{target}-\right.\right.$ Sample $\left.\mathrm{Ct}_{\text {miR-16 }}\right)$ - $\left(\right.$ Control $\mathrm{Ct}_{\text {target }}-$ Control $\left.\left.\mathrm{Ct}_{\text {miR-16 }}\right)\right]$.

Statistical analysis. After the homogeneity test of variance was verified, the differences between the groups were analyzed using one-way ANOVA, post hoc multiple comparisons LSD test. The reproducibility of RNA extraction and microRNA detection was analyzed by linear correlation. AUC-ROC with $95 \%$ confidence interval (95\% CI) were established for microRNAs in discriminating the different subject groups. The relationships of the microRNAs with the patient clinical characteristics were analyzed by Spearman for categorical items or Pearson for quantitative data. All data analyses were conducted with SPSS software (version 11.0). All P-values were two-sided, and a P-value of less than 0.05 was considered to indicate statistical significance.

\section{Results}

Reproducibility of extraction and detection offecalmicroRNAs. Clinical and demographical data of the patients are presented in Table I. RNA concentrations in the stool samples ranged from 386.3 to $3,399.9 \mathrm{ng} / \mu \mathrm{l}$. To assess the reproducibility of the methodology for microRNA extraction and detection, we repeated these procedures in a subset of 6 stool samples from three $\mathrm{PCa}$ and three $\mathrm{CP}$ patients at 6-month intervals. As showed in Fig. 1, there was a high linear correlation in amounts of the fecal total-RNA $\left(\mathrm{R}^{2}=0.998, \mathrm{P}<0.001\right.$, Fig. $\left.1 \mathrm{~A}\right)$ and miR-16 detection $\left(\mathrm{R}^{2}=0.988, \mathrm{P}<0.001\right.$, Fig. $\left.1 \mathrm{~B}\right)$ between the two independent extraction procedures, indicating that the 


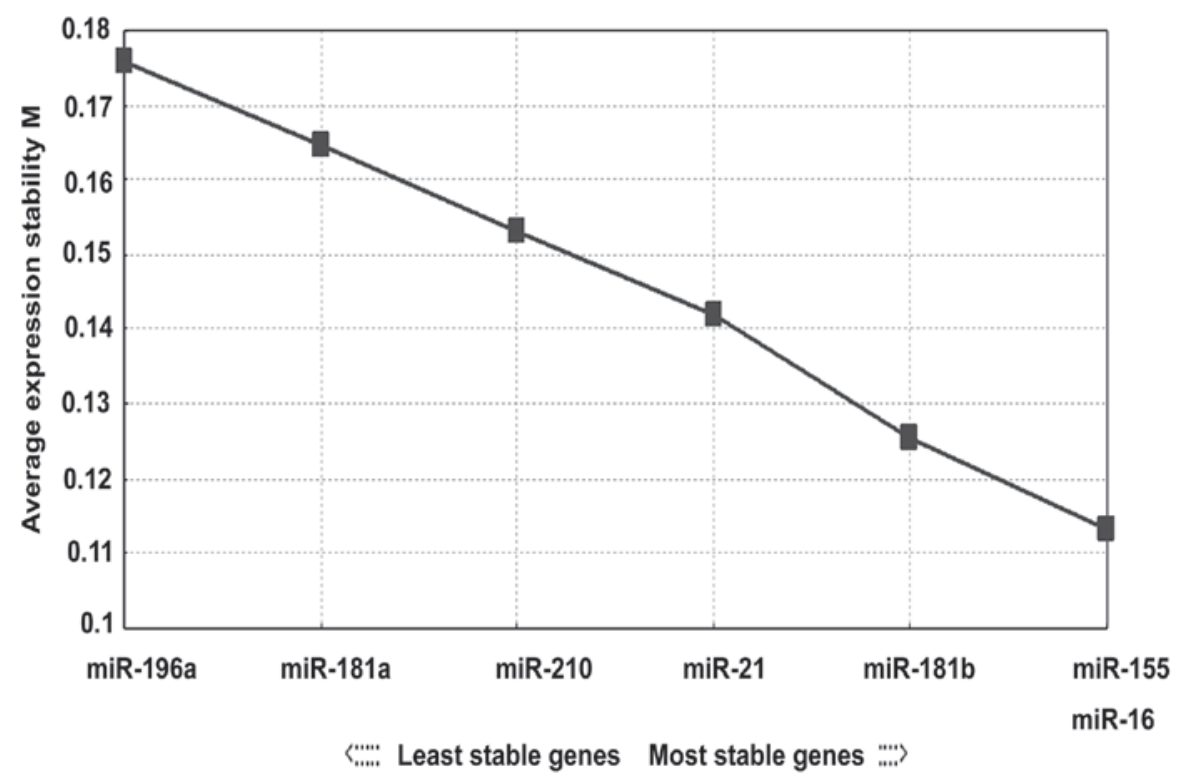

Figure 2. Validation of miR-16 as a normalizer by Genorm software, by which the most stably expressed genes were determined after input of all Ct data of the participant genes in all samples.

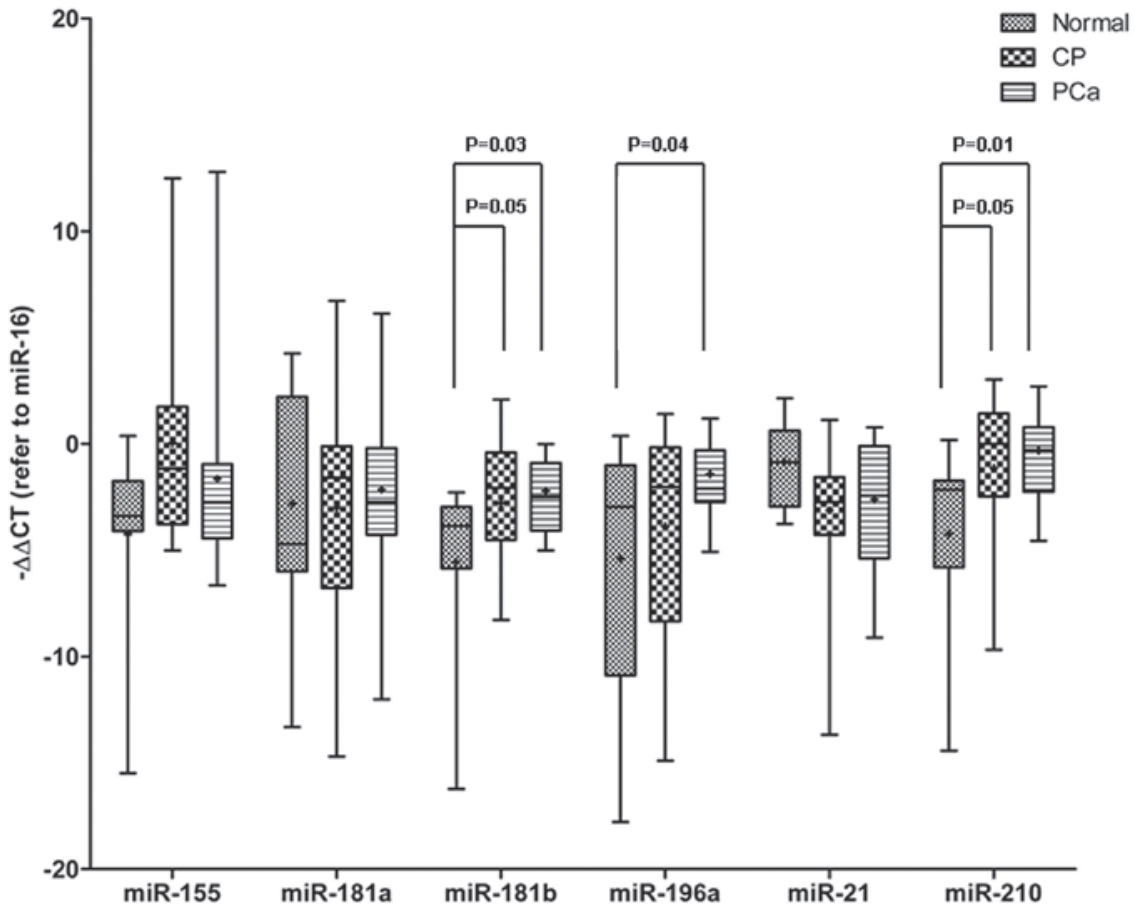

Figure 3. Box-plot of the relative abundances of fecal miR-21, miR-155, miR-181a, miR-181b, miR-196a and miR-210 in the stool samples from healthy staff members $(n=13)$, patients with $\mathrm{CP}(\mathrm{n}=22)$ and pancreatic cancer $(\mathrm{PCa})(\mathrm{n}=29)$. The upper and lower limits of the boxes and the lines across the boxes indicate the 75th and 25th percentiles and median, respectively. Error bars indicate the 90th and 10th percentiles. After the homogeneity tests of variances were equal in the three groups, the differences among the groups were analyzed using one-way ANOVA: post hoc multiple comparisons LSD test.

methods for the fecal total-RNA extraction and detection were highly reproducible.

Comparison of microRNA profiles in stool samples among the three groups of subjects. As showed in Fig. 2, miR-16 was validated as a normalizer by Genorm software. With miR-16 as a normalizer, the relative abundances of miR-181b, miR-196a,
miR-210, miR-155, miR-181a and miR-21 were determined. It was observed that the relative abundances of miR-181b, miR196a and miR-210 were significantly greater in the PCa group than in the normal group $(\mathrm{P}=0.033, \mathrm{P}=0.043$ and $\mathrm{P}=0.011$, respectively) (Fig. 3). In addition, the relative abundances of miR-181b and miR-210 appeared to be greater in the CP group than in the normal group although the difference was not statis- 
Table II. Receiver operating characteristic analysis of the relative abundances of fecal miR-181b, miR-196a and miR-210 for differentiating among patients with pancreatic cancer (PCa), those with chronic pancreatitis (CP) and normal subjects.

Relative abundance

(reference to miR-16)

Patient group

$95 \%$ Confidence interval

\begin{tabular}{|c|c|c|c|c|c|}
\hline \multirow[t]{4}{*}{ miR-181b } & PCa vs. normal & 0.745 & 0.012 & 0.597 & 0.894 \\
\hline & CP vs. normal & 0.780 & 0.004 & 0.627 & 0.933 \\
\hline & $\mathrm{PCa}+\mathrm{CP}$ vs. normal & 0.760 & 0.004 & 0.639 & 0.882 \\
\hline & PCa vs. CP & 0.451 & 0.555 & 0.286 & 0.617 \\
\hline \multirow[t]{4}{*}{ miR-196a } & PCa vs. normal & 0.618 & 0.226 & 0.421 & 0.815 \\
\hline & CP vs. normal & 0.549 & 0.633 & 0.349 & 0.749 \\
\hline & $\mathrm{PCa}+\mathrm{CP}$ vs. normal & 0.588 & 0.329 & 0.408 & 0.769 \\
\hline & PCa vs. CP & 0.539 & 0.634 & 0.370 & 0.708 \\
\hline \multirow[t]{4}{*}{$\operatorname{miR}-210$} & PCa vs. normal & 0.772 & 0.005 & 0.629 & 0.914 \\
\hline & CP vs. normal & 0.766 & 0.009 & 0.610 & 0.922 \\
\hline & $\mathrm{PCa}+\mathrm{CP}$ vs. normal & 0.769 & 0.003 & 0.647 & 0.891 \\
\hline & PCa vs. CP & 0.464 & 0.662 & 0.297 & 0.630 \\
\hline
\end{tabular}

${ }^{a}$ The nonparametric Mann-Whitney U test was used to compare the patients with pancreatic diseases and normal controls.
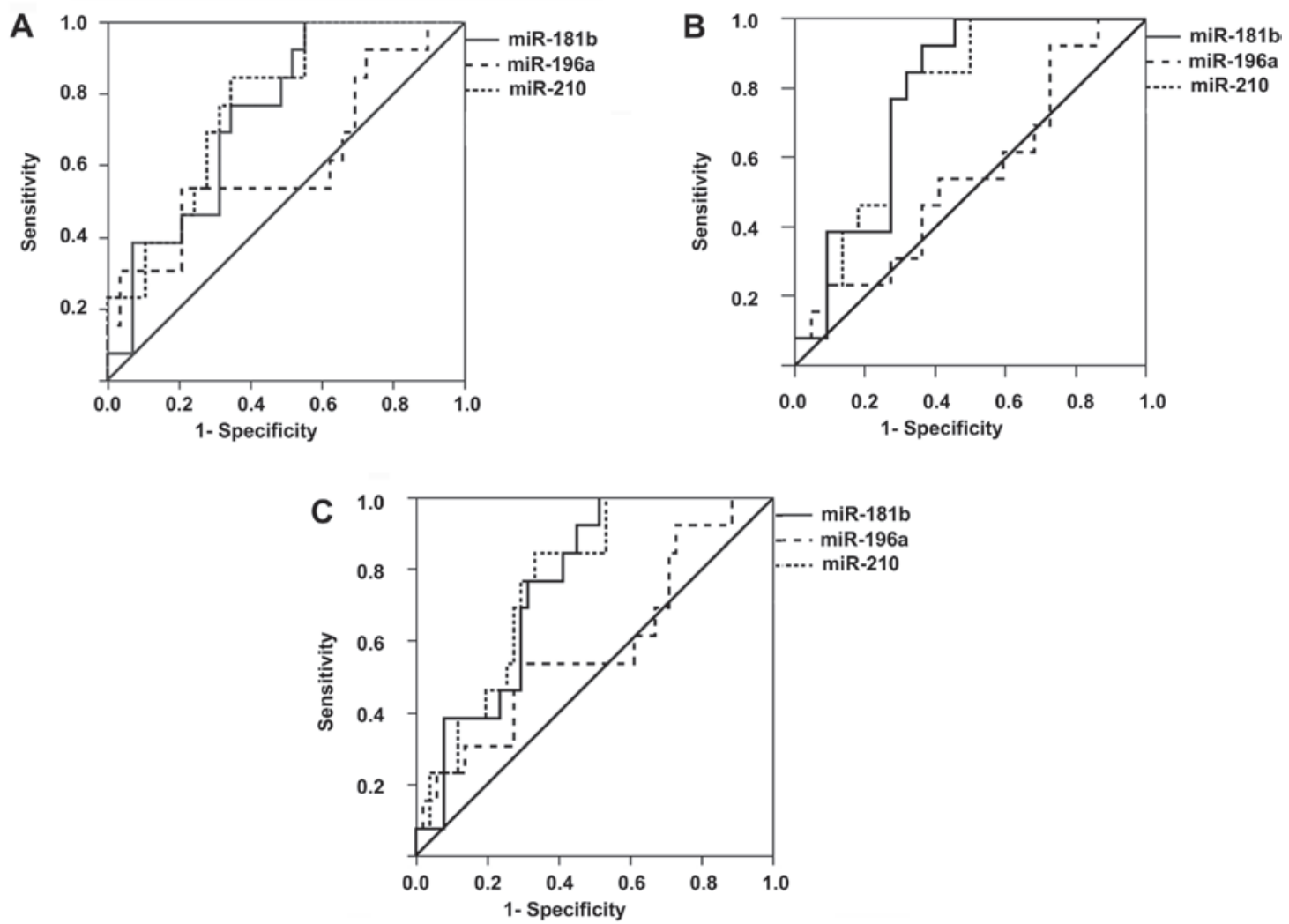

Figure 4. Diagnostic performance of the relative abundances of fecal miR-181b, miR-196a and miR-210. (A) AUC-ROC for differentiating PCa patients from normal subjects (miR-181b, 0.745, $\mathrm{P}=0.012$; miR-196a, 0.618, $\mathrm{P}=0.555$; miR-210, 0.772, $\mathrm{P}=0.005$ ). (B) AUC-ROC for differentiating $\mathrm{CP}$ patients from normal subjects (miR-181b, 0.780, $\mathrm{P}=0.004$; miR-196a, 0.633, $\mathrm{P}=0.349$; miR-210, 0.766, $\mathrm{P}=0.009$ ). (C) AUC-ROC for differentiating patients with pancreatic diseases ( $\mathrm{PCa}$ and $\mathrm{CP}$ ) from normal subjects (miR-181b, 0.760, $\mathrm{P}=0.004$; miR-196a, 0.588, $\mathrm{P}=0.329$; miR-210, 0.769, $\mathrm{P}=0.003$ ).

tically significant $(\mathrm{P}=0.088$ and $\mathrm{P}=0.053$, respectively); there was no difference between the PCa and CP groups. Concerning miR-155, miR-181a and miR-21, no differences in their relative abundances were also found among the three groups.
Diagnostic performance of fecal microRNAs. As showed in Table II and Fig. 4, the ability of miR181b, miR-196a and miR-210 to diagnose different pancreatic diseases was evaluated by ROC curves. Only miR181b and miR-210 had 
Table III. Correlations between the relative abundances of microRNAs and clinical features in patients with pancreatic cancer (PCa).

\begin{tabular}{|c|c|c|c|c|c|c|c|}
\hline \multirow[b]{2}{*}{ Characteristics } & \multirow{2}{*}{$\begin{array}{l}\text { Cases } \\
(n=29)\end{array}$} & \multicolumn{2}{|c|}{$\operatorname{miR}-181 b$} & \multicolumn{2}{|c|}{ miR-196a } & \multicolumn{2}{|c|}{ miR-210 } \\
\hline & & mean $\pm \mathrm{SD}$ & P-value (r) & mean $\pm \mathrm{SD}$ & P-value (r) & mean $\pm \mathrm{SD}$ & P-value (r) \\
\hline Gender & & & $0.419(0.156)$ & & $0.530(0.121)$ & & $0.893(0.026)$ \\
\hline Male & 19 & $0.86 \pm 5.18$ & & $1.52 \pm 6.79$ & & $0.43 \pm 5.31$ & \\
\hline Female & 10 & $2.94 \pm 2.39$ & & $1.37 \pm 3.97$ & & $0.72 \pm 2.81$ & \\
\hline Age (years) & & & $0.091(0.320)$ & & $0.966(0.008)$ & & $0.093(0.318)$ \\
\hline$<60$ & 9 & $3.56 \pm 2.39$ & & $1.55 \pm 1.62$ & & $1.42 \pm 2.55$ & \\
\hline$\geq 60$ & 20 & $1.62 \pm 3.99$ & & $1.36 \pm 5.97$ & & $0.17 \pm 4.21$ & \\
\hline Weight (kg) & & & $0.455(0.353)$ & & $0.915(0.022)$ & & $0.730(0.071)$ \\
\hline$<60$ & 16 & $1.58 \pm 4.81$ & & $0.91 \pm 4.33$ & & $0.54 \pm 4.76$ & \\
\hline$\geq 60$ & 13 & $2.79 \pm 2.32$ & & $0.64 \pm 4.79$ & & $1.36 \pm 3.27$ & \\
\hline $\begin{array}{l}\text { Smoking index } \\
\text { (cigarettes/year) }\end{array}$ & 21 & & $0.549(0.311)^{\mathrm{a}}$ & & $0.387(0.436)^{\mathrm{a}}$ & & $0.853(0.098)^{\mathrm{a}}$ \\
\hline $\begin{array}{l}\text { Tumor maximum } \\
\text { diameter }(\mathrm{cm})\end{array}$ & 29 & & $0.117(0.408)^{\mathrm{a}}$ & & $0.041(0.516)$ & & $0.850(0.052)^{\mathrm{a}}$ \\
\hline $\begin{array}{l}\text { Pancreatic duct } \\
\text { diameter }(\mathrm{cm})\end{array}$ & 29 & & $0.702(0.162)^{\mathrm{a}}$ & & $0.923(0.041)^{\mathrm{a}}$ & & $0.914(0.046)^{\mathrm{a}}$ \\
\hline \multicolumn{8}{|l|}{ CA19-9 (U/1) } \\
\hline$<37$ & 7 & $3.36 \pm 2.75$ & $0.530(0.121)$ & $2.15 \pm 1.36$ & $0.724(0.068)$ & $1.51 \pm 2.94$ & $0.822(0.044)$ \\
\hline$\geq 37$ & 22 & $1.86 \pm 3.88$ & & $1.59 \pm 5.71$ & & $0.06 \pm 4.02$ & \\
\hline CEA ( ng/ml) & & & $0.757(0.060)$ & & $0.649(0.088)$ & & $0.437(0.150)$ \\
\hline$<10$ & 19 & $2.24 \pm 4.34$ & & $1.20 \pm 6.09$ & & $0.14 \pm 4.57$ & \\
\hline$\geq 10$ & 10 & $2.19 \pm 1.91$ & & $1.83 \pm 1.81$ & & $0.66 \pm 1.69$ & \\
\hline Bilirubin $(\mu \mathrm{mol} / \mathrm{l})$ & & & $0.986(0.003)$ & & $0.208(0.241)$ & & $0.503(0.130)$ \\
\hline$<17.1$ & 17 & $177 \pm 4.62$ & & $0.09 \pm 5.28$ & & $0.06 \pm 4.83$ & \\
\hline$\geq 17.1$ & 12 & $2.87 \pm 1.44$ & & $3.31 \pm 4.06$ & & $0.70 \pm 1.62$ & \\
\hline ALT ( U/l) & & & $0.079(0.332)$ & & $0.203(0.527)$ & & $0.676(0.081)$ \\
\hline$<40$ & 19 & $1.17 \pm 4.29$ & & $0.19 \pm 4.99$ & & $0.22 \pm 4.59$ & \\
\hline$\geq 40$ & 10 & $3.21 \pm 1.68$ & & $3.75 \pm 4.31$ & & $0.51 \pm 1.63$ & \\
\hline AST (U/l) & & & $0.230(0.230)$ & & $0.103(0.479)$ & & $0.617(0.097)$ \\
\hline$<40$ & 19 & $1.66 \pm 4.29$ & & $0.32 \pm 5.09$ & & $0.43 \pm 4.64$ & \\
\hline$\geq 40$ & 10 & $3.29 \pm 1.62$ & & $3.51 \pm 4.29$ & & $0.11 \pm 1.39$ & \\
\hline r-GT (U/l) & & & $0.071(0.372)$ & & $0.850(0.447)$ & & $0.434(0.151)$ \\
\hline$<50$ & 13 & $0.87 \pm 4.76$ & & $0.83 \pm 5.72$ & & $0.04 \pm 5.37$ & \\
\hline$\geq 50$ & 16 & $3.32 \pm 1.96$ & & $3.24 \pm 3.54$ & & $0.61 \pm 1.94$ & \\
\hline WBC (x109/1) & & & $0.752(0.061)$ & & $0.377(0.170)$ & & $0.645(0.089)$ \\
\hline$<10$ & 24 & $2.35 \pm 3.91$ & & $2.01 \pm 4.53$ & & $0.63 \pm 3.96$ & \\
\hline$\geq 10$ & 5 & $1.63 \pm 2.19$ & & $1.40 \pm 6.72$ & & $1.15 \pm 2.82$ & \\
\hline Blood sugar (mmol/l) & & & $0.477(0.138)$ & & $0.427(0.153)$ & & $0.09(0.318)$ \\
\hline$<6.1$ & 18 & $2.33 \pm 4.29$ & & $1.07 \pm 6.18$ & & $0.99 \pm 2.55$ & \\
\hline$\geq 6.1$ & 11 & $2.04 \pm 2.40$ & & $199 \pm 2.11$ & & $1.09 \pm 0.06$ & \\
\hline T stage & 29 & & $0.595(0.114)$ & & $0.255(0.242)$ & & $0.916(0.023)$ \\
\hline $\mathrm{N}$ stage & 29 & & $0.649(0.098)$ & & $0.156(0.299)$ & & $0.417(0.174)$ \\
\hline M stage & 29 & & $0.801(0.054)$ & & $0.274(0.232)$ & & $0.245(0.247)$ \\
\hline Clinical stage & 29 & & $0.642(0.102)$ & & $0.628(0.107)$ & & $0.580(0.122)$ \\
\hline
\end{tabular}

aPearson correlation analysis; Spearman rank correlation analysis was used for other comparisons. CA19-9, carbohydrate antigen 19-9; CEA, carcinoma embryonic antigen; ALT, alanine aminotransferase; AST, aspartate aminotransferase; r-GT, glutamyl transpeptidase; WBC, white blood count. 
significant powers to differentiate among the three different groups. For differentiating PCa patients from normal subjects, the AUC-ROC of miR-181b and miR-210 were 0.745 (95\% CI: $0.597-0.894)$ and 0.772 (95\% CI: 0.629-0.914), with the sensitivity and specificity of miR-181b (cut-off value $=2.52$ ) and miR-210 (cut-off value $=1.54$ ) of 84.6 and $51.7 \%$, and 84.6 and $65.5 \%$, respectively. For differentiating $\mathrm{CP}$ patients from normal subjects, the AUC-ROC of miR-181b and miR-210 were 0.780 (95\% CI: $0.627-0.933)$ and 0.766 (95\% CI: $0.610-0.922$ ), with the sensitivity and specificity of miR-181b (cut-off value $=2.52$ ) and miR-210 (cut-off value $=1.59$ ) of 84.6 and $58.8 \%, 84.6$ and $66.7 \%$, respectively. For differentiating patients with pancreatic diseases (i.e. PCa and $\mathrm{CP}$ ) from normal subjects, the AUC-ROC of miR-181b and miR-210 were 0.760 (95\% CI: 0.639-0.882) and 0.769 (95\% CI: $0.647-0.891)$, with the sensitivity and specificity of miR-181b (cut-off value $=3.11$ ) and miR-210 (cut-off value $=1.59$ ) of 76.9 and $72.7 \%, 84.6$ and $69.2 \%$, respectively.

Correlation of fecal microRNAs with clinical characteristics of the PCa patients. Table III shows the correlations between the relative abundances of miR-181b, miR-196a and miR-210 and clinical characteristics of the PCa patients. The clinical data included patient age, gender, weight, smoking index, the maximum diameter of the tumor, the diameter of the pancreatic duct, TNM stage, clinical stage, the levels of serum CEA, CA19-9, ALT, AST, r-GT, bilirubin, WBC and fasting blood glucose. Only a positive correlation between miR-196a and the maximum diameter of the tumor was observed (Spearman $\mathrm{r}=0.516, \mathrm{P}=0.041)$. No significant correlations were found between miR-181b or miR-210 and any clinical characteristics.

\section{Discussion}

In recent years, several studies have shown that certain microRNAs are aberrantly expressed in human malignancies (26), indicating that a new class of cancer biomarkers could be explored for early diagnosis of cancers. Here, the present study showed that aberrant expression of microRNAs in stool samples was a potential biomarker for the screening and diagnosis of PCa. First, it was demonstrated that the methodology used for the preparation of the microRNAs from stool samples, even those stored in a freezer for up to 2 years, was feasible, convenient and reproducible, and the amounts of microRNAs detected by TaqMan PCR between intra-individuals was consistent. Second, fecal miR-181b, miR-196a and miR-210 were identified to have significant power in differentiating PCa patients from normal subjects, although their ability in discriminating PCa from CP was not found in this study. These findings showed the potential value of microRNAs in stool samples as a new biomarker for PCa screening.

Several studies have shown that unique microRNA expression profiles are present in a number of human cancers such as cancers of the breast, lung, esophagus, prostate and pancreas, and differential expression profiles of microRNAs correlate with important histopathological features including tumor stage, proliferative capacity and vascular invasion (27). With regard to PCa, varying expression profiles of a number of microRNAs distinguishing malignant lesions from normal pancreatic tissue and $\mathrm{CP}$ have also been reported
$(17,28,29)$. However, none of these varying expression profiles of microRNAs has been detected in stool samples. In the present study, a panel of six microRNAs (miR-21, miR-155, miR-181a, miR-181b, miR-196a and miR-210) was selected to investigate their expression in the stool samples. As a result, only miR-181b, miR-196a and miR-210 showed significantly aberrant expression in stool of PCa patients, compared with that in the stool of normal subjects.

These microRNAs were selected in our study according to findings in previous studies (17,24,25,28,30-33). Earlier, Lee et al (25) reported that the expression of mature miR-21, miR-155, miR-181a and their precursors was higher in $\mathrm{PCa}$ tissues than in adjacent benign tissues and normal pancreas specimens. Roldo et al (17) reported that the overexpression of miR-21 was strongly associated with both an increased Ki67 proliferation index and liver metastasis of PCa, and Dillhoff et al (24) showed that miR-21 was a potential predictor for poor survival of node-negative PCa patients. Bloomston et al (28) observed that, among the six microRNAs included in the present study, five (miR-21, miR-155, miR-181a, miR-181b and miR-210) were upregulated in PCa tissues compared with matched adjacent benign tissues and CP tissues but miR-196a did not help differentiate PCas from normal pancreas or $\mathrm{CP}$ while miR-196a as a predictor for survival and progression was proposed by studies of Zhang et al (33) and Szafranska et al (32). Furthermore, Greither et al (30) reported that the expression levels of miR-155 and miR-210 were higher in pancreatic tumor tissues compared to normal tissues and significantly correlated with poorer overall survival. Recently, Mardin et al (31), in a relevant review of PCa tissue-specific microRNAs, recommended that the six selected target microRNAs, which were overexpressed in PCa tissues vs. normal tissues and $\mathrm{CP}$ tissues, were to be further evaluated as targets for diagnosis and therapeutics.

It is noted in the present study that the fecal microRNA signature was not fully consistent with that of solid tumors. In the present study, only miR-181b, miR-196a and miR-210 expression levels were significantly higher in the stool of the PCa group compared with the normal group. A similar result was also observed in our previous study (34), in which eight PCa tissue-specific microRNAs (miR-155, miR-16, miR-181a, miR-181b, miR-21, miR-196a, miR-222 and miR-221) were detected in the serum samples of PCa patients, but only three microRNAs (miR-21, miR-155 and miR-196a) were found to be overexpressed in patients with pancreatic diseases (including PCa and $\mathrm{CP}$ ) compared with normal subjects. We hypothesize that there are two sources for microRNAs in stool samples: one from the tumor cells exfoliated from the tumor masses which are leaked into the digestive tract along with the pancreatic juice, and another from the tumor-derived exosomes in circulating blood which can be secreted into the digestive tract along with the gastrointestinal secretions with an active (selective) process. It is probably that this active (selective) process causes the discrepancy in the expression of microRNA between tissues and stool samples. This hypothesis was proposed by Taylor and Gercel-Taylor (35) who observed that the tumor-derived exosomes in the blood of ovarian cancer patients contained the majority of the cancer-specific microRNA, while some microRNAs were uniquely elevated in the exosomes. These authors explained that there was compart- 
mentalization of microRNAs into exosomes and/or an active (selective) process in producing tumor-derived exosomes.

Another issue deserved to be mentioned is the selection of miR-16 as the normalizer in the present study. Previously, Link et al (21) successfully used miR-16 as a normalizer to screen and identify fecal microRNAs as potential biomarkers for colorectal cancer. microRNAs were also recommended as a normalizer by Genorm software (http://medgen.ugent.be/ genorm) (18), which can calculate the gene expression stability to determine the most stable reference genes, and be regarded as the de facto standard with more than 2000 citations (cited by Google Scholar, September 2009).

The potential of fecal microRNAs as biomarkers for detecting PCa was evaluated through diagnostic characteristics although a small number of clinical samples were analyzed in this study. For differentiating the patients with pancreatic diseases (PCa and CP) from normal subjects, miR-181b and miR-210 had significant value, while miR-196a did not. However, in the present study, all three microRNAs failed to show power for differentiating PCa from $\mathrm{CP}$, which might be due to the small number of subjects enrolled in this study. Currently, a larger study is ongoing in our department to specifically assess the ability of microRNAs for differentiating PCa from CP.

After analysis of the correlations of the three microRNAs with the clinical characteristics of the PCa cases, miR-196a was found to be positively correlated with the maximum diameter of the tumor. Previously, studies only showed that the relative abundance of miR-196a $(28,33)$ in sera of PCa patients (34) was a predictor of poor survival; however, the correlations between miR-196a and other clinical characteristic were not investigated. Thus, our novel observation on the correlation of fecal miR-196a with the tumor maximum diameter warrants further studies exploring the underlying mechanisms. More importantly, the significant overexpression of fecal miR-181b and miR-210 in PCa patients indicates that these microRNAs are new candidate biomarkers for the screening of early $\mathrm{PCa}$ evolution.

In conclusion, the methodology for the extraction and detection of fecal microRNAs is easy and reproducible. The fact that some microRNAs that are highly expressed in $\mathrm{PCa}$ tissues are not consistently expressed in stool samples suggests that the fecal cancer-specific microRNAs may be produced by secretions of digestive tract cells by an active (selective) process through tumor-derived exosomes. Moreover, miR-181b and miR-210 are increasingly expressed in the stool of patients with PCa compared with normal individuals, suggesting that these microRNAs may have potential to be used as new potential biomarkers for PCa screening.

\section{Acknowledgements}

The present study was supported by a grant (2006BAI02A12, 30910103911) from the NSFC of China and the Key Scientific and Technological Project of Shanghai (No. 11441901800). The authors also would like to thank SBC, Oebiotech and Biotecan for their technical support.

\section{References}

1. Real FX: A 'catastrophic hypothesis' for pancreas cancer progression. Gastroenterology 124: 1958-1964, 2003.
2. Wade TP, Halaby IA, Stapleton DR, Virgo KS and Johnson FE: Population-based analysis of treatment of pancreatic cancer and Whipple resection: Department of Defense Hospitals, 1989-1994. Surgery 120: 680-687, 1996.

3. Bartel DP: MicroRNAs: genomics, biogenesis, mechanism, and function. Cell 116: 281-297, 2004.

4. Carrington JC and Ambros V: Role of microRNAs in plant and animal development. Science 301: 336-338, 2003.

5. Lim LP, Glasner ME, Yekta S, Burge CB and Bartel DP: Vertebrate microRNA genes. Science 299: 1540, 2003.

6. Lim LP, Lau NC, Weinstein EG, et al: The microRNAs of Caenorhabditis elegans. Genes Dev 17: 991-1008, 2003.

7. Ambros V: microRNA pathways in flies and worms: growth, death, fat, stress, and timing. Cell 113: 673-676, 2003.

8. Ke XS, Liu CM, Liu DP and Liang CC: microRNAs: key participants in gene regulatory networks. Curr Opin Chem Biol 7: 516-523, 2003.

9. Bartel B and Bartel DP: microRNAs: at the root of plant development? Plant Physiol 132: 709-717, 2003.

10. Chen X, Ba Y, Ma L, et al: Characterization of microRNAs in serum: a novel class of biomarkers for diagnosis of cancer and other diseases. Cell Res 18: 997-1006, 2008.

11. Hui AB, Shi W, Boutros PC, et al: Robust global microRNA profiling with formalin-fixed paraffin-embedded breast cancer tissues. Lab Invest 89: 597-606, 2009.

12. Mitchell PS, Parkin RK, Kroh EM, et al: Circulating microRNAs as stable blood-based markers for cancer detection. Proc Natl Acad Sci USA 105: 10513-10518, 2008.

13. Lu J, Getz G, Miska EA, et al: microRNA expression profiles classify human cancers. Nature 435: 834-838, 2005.

14. He H, Jazdzewski K, Li W, et al: The role of microRNA genes in papillary thyroid carcinoma. Proc Natl Acad Sci USA 102: 19075-19080, 2005.

15. Iorio MV, Ferracin M, Liu CG, et al: microRNA gene expression deregulation in human breast cancer. Cancer Res 65: 7065-7070, 2005 .

16. Murakami Y, Yasuda T, Saigo K, et al: Comprehensive analysis of microRNA expression patterns in hepatocellular carcinoma and non-tumorous tissues. Oncogene 25: 2537-2545, 2006.

17. Roldo C, Missiaglia E, Hagan JP, et al: microRNA expression abnormalities in pancreatic endocrine and acinar tumors are associated with distinctive pathologic features and clinical behavior. J Clin Oncol 24: 4677-4684, 2006.

18. Vandesompele J, De Preter K, Pattyn F, et al: Accurate normalization of real-time quantitative RT-PCR data by geometric averaging of multiple internal control genes. Genome Biol 3: RESEARCH0034, 2002.

19. Yanaihara N, Caplen N, Bowman E, et al: Unique microRNA molecular profiles in lung cancer diagnosis and prognosis. Cancer Cell 9: 189-198, 2006.

20. Hanke M, Hoefig K, Merz H, et al: A robust methodology to study urine microRNA as tumor marker: microRNA-126 and microRNA-182 are related to urinary bladder cancer. Urol Oncol 28: 655-661, 2010.

21. Link A, Balaguer F, Shen Y, et al: Fecal microRNAs as novel biomarkers for colon cancer screening. Cancer Epidemiol Biomarkers Prev 19: 1766-1774, 2010.

22. Kawarada Y: New classification of pancreatic carcinoma apan Pancreas Society. Nihon Shokakibyo Gakkai Zasshi 100: 974-980, 2003 (In Japanese).

23. Bhatt K, Zhou L, Mi QS, Huang S, She JX and Dong Z: microRNA-34a is induced via p53 during cisplatin nephrotoxicity and contributes to cell survival. Mol Med 16: 409-416, 2010.

24. Dillhoff M, Liu J, Frankel W, Croce C and Bloomston M: microRNA-21 is overexpressed in pancreatic cancer and a potential predictor of survival. J Gastrointest Surg 12: 2171-2176, 2008.

25. Lee EJ, Gusev Y, Jiang J, et al: Expression profiling identifies microRNA signature in pancreatic cancer. Int J Cancer 120: 1046-1054, 2007.

26. Volinia S, Calin GA, Liu CG, et al: A microRNA expression signature of human solid tumors defines cancer gene targets. Proc Natl Acad Sci USA 103: 2257-2261, 2006.

27. Lynam-Lennon N, Maher SG and Reynolds JV: The roles of microRNA in cancer and apoptosis. Biol Rev Camb Philos Soc 84: 55-71, 2009.

28. Bloomston M, Frankel WL, Petrocca F, et al: microRNA expression patterns to differentiate pancreatic adenocarcinoma from normal pancreas and chronic pancreatitis. JAMA 297: 1901-1908, 2007. 
29. Szafranska AE, Davison TS, John J, et al: microRNA expression alterations are linked to tumorigenesis and non-neoplastic processes in pancreatic ductal adenocarcinoma. Oncogene 26 : 4442-4452, 2007

30. Greither T, Grochola LF, Udelnow A, Lautenschlager C, Wurl P and Taubert H: Elevated expression of microRNAs 155, 203, 210 and 222 in pancreatic tumors is associated with poorer survival. Int J Cancer 126: 73-80, 2010

31. Mardin WA and Mees ST: microRNAs: novel diagnostic and therapeutic tools for pancreatic ductal adenocarcinoma? Ann Surg Oncol 16: 3183-3189, 2009.

32. Szafranska AE, Doleshal M, Edmunds HS, et al: Analysis of microRNAs in pancreatic fine-needle aspirates can classify benign and malignant tissues. Clin Chem 54: 1716-1724, 2008.
33. Zhang Y, Li M, Wang $\mathrm{H}$, et al: Profiling of 95 microRNAs in pancreatic cancer cell lines and surgical specimens by real-time PCR analysis. World J Surg 33: 698-709, 2009.

34. Kong X, Du Y, Wang G, et al: Detection of differentially expressed microRNAs in serum of pancreatic ductal adenocarcinoma patients: miR-196a could be a potential marker for poor prognosis. Dig Dis Sci 56: 602-609, 2011.

35. Taylor DD and Gercel-Taylor C: microRNA signatures of tumor-derived exosomes as diagnostic biomarkers of ovarian cancer. Gynecol Oncol 110: 13-21, 2008. 